\title{
A SUPER-SENSITIVE SENSOR INTRUDER ALARM SYSTEM
}

This paper was downloaded from TechRxiv (https://www.techrxiv.org).

\section{LICENSE}

CC BY 4.0

SUBMISSION DATE / POSTED DATE

24-04-2021 / 28-04-2021

\section{CITATION}

singh, Shaurya (2021): A SUPER-SENSITIVE SENSOR INTRUDER ALARM SYSTEM. TechRxiv. Preprint. https://doi.org/10.36227/techrxiv.14479206.v1

DOI 


\section{A SUPER-SENSITIVE SENSOR INTRUDER ALARM SYSTEM}

\author{
Shaurya Pratap Singh \\ 19SECE1030014 \\ $2^{\mathrm{ND}}$ YEAR, ECE \\ School of electronics and \\ communication engineering \\ Galgotias University \\ Greater Noida India
}

\author{
Abhay Kumar Shah \\ 19SECE1030030 \\ $2^{\mathrm{ND}}$ YEAR, ECE \\ School of electronics and \\ communication engineering \\ Galgotias University \\ Greater Noida India
}

\begin{abstract}
A super sensitive intruder circuit is refined to mindful the individual when an outcast attempt to go into the house. In the event that there is a hindrance in front of the IR sensor, it delivers an intrude signal. This interruption signal is given to the speaker to alert the individual . The IR transmitter consistently produces IR (infrared) beams that the IR recipient needs to get. When there is an obstacle in front of the IR sensor, the communicated IR beams are reflected in the IR collector. At the point when the IR beneficiary gets reflected IR beams, the yield of Op-Amp is high. This yield of the op-amp is associated with the reset pin of the 555 timer.

Keywords-IR SENSOR, IC LM358, 555 TIMER
\end{abstract}

\section{INTRODUCTION}

The requirement for security is the fundamental necessity of any individual. The inclination that we are protected and everything around us is vital for a safe life. In any case, in this shaky world, when wrongdoing activity, and threat are at peak, how can a sense of security be attained? Here, the super sensitive intruder alarm system furnishes us with an answer and therefore, an ever increasing number of individuals are introducing them to remain free from any danger. Different electronic security frameworks can be utilized at home and other significant working environments for wellbeing and security purposes.

A security alarm system is a device intended to unauthorizedly identify interruption into a structure or different regions, like a home or school. Security alerts are utilized for burglary (robbery) or property harm in private, business, mechanical, and military property and individual insurance against an outcast. Security alarms in residential areas show a correlation with less theft. Similarly, car security alarm system help ensure vehicles and their substance.

Some security alarm system fill a similar need of assurance by consolidating robbery, giving protection to fire and interruption. Intrusion alarm systems can be combined with closed-circuit television surveillance (CCTV) systems to automatically record outsider movements and interface to access control systems for electrically closed entrances. Systems range from little, independent noisemakers to complex, multi-facet frameworks with PC observing and control. It might likewise incorporate a two-way voice that permits correspondence between the panel and the monitoring station.

\section{LITERATURE SURVEY}

\section{Design and Implementation of Smart Home-Based Security System}

This paper recommends home security using two techniques. The first webcam and the second International Journal of Trend in Scientific Research and Development (IJTSRD). At whatever point the speed or movement is before a web camera, in the first technique utilizing GSM innovation, it gives yield to the controller and the alarm is turned on. In the subsequent strategy, the GSM method is utilized. GSM is an innovation that communicates something specific as per the sign gave to it and an warning message is shipped to all numbers that are saved in the SIM card.

\section{An analysis on Home Automation System Based on Internet of Things}

An low cost smart home system, which utilizes Android applications to speak with the cloud and provide switching functions, is advertised. The framework wipes out the utilization of (PCs) and other PC peripherals which leads a general reduction in the expense of the system. Unlike similar systems that utilize Bluetooth module networks, the proposed system utilizes the Internet of Things (IoT) to distantly control electrical/electronic gadgets. Electrical/electronic gear switches are coordinated into the system to exhibit the adequacy and possibility of the framework.

\section{EXISTING SYSTEM}
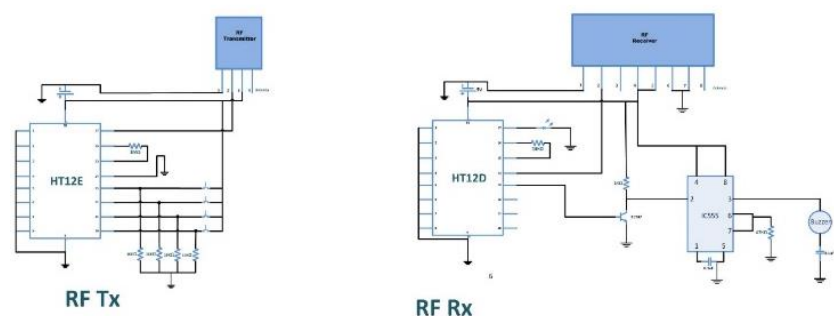


\section{"Wireless industrial outsider alarm system"}

The project uses LDR sensors to sense the foreigner. When the LDR sensor value changes state (from low resistance to high resistance), the encoder data pin receives logic 1 (high) input. It transmits to the receiver via the transmitter module $(434 \mathrm{MHz})$. When the decoder data pin becomes high, it triggers IC555 and triggers an alarm.

\section{Working:}

1. The basic principle rule of this device is to trigger the IC555 timer to activate the buzzer when the LDR resistance is high.

2. The LDR resistance changes as indicated by the light falling on it. Resistance increments when it projects a shadow. In view of this occurrence, here we are activating the alarm.

3. In this project this circuit is partitioned into two sections, LDR control on the transmitter side and buzzer control on the receiver side.

4. At the point when the LDR projects a shadow, its resistance expands, which changes the logic state at the input of the encoder HT12E. So it transmits this information to the receiver through the $434 \mathrm{MHz}$ ASK TX module

5. The $434 \mathrm{MHz}$ ASK RX module on the receiver side gets this signal. It restores this to the decoder HT12D. The HT12D pin gives a higher output on the same pin that is associated with the alarm activating circuit.

6. Transistor gets high information so it operates IC555 and gives low trigger information

7. The IC555 produces a high output pulse that buzzers for a couple of moments. Duration relies upon the associated RC components.

8. The benefit of this project is that we can put the transmitter anyplace within the range, and numerous alarms can be set with so many guards. We can initiate numerous receivers with only one transmitter.

\section{PROPOSED SYSTEM}

\section{Working principle}

The IR transmitter always emits IR (infrared) rays that the IR receiver has to receive. When there is an obstacle in front of the IR sensor, the transmitted IR rays are reflected in the IR receiver. When the IR receiver receives reflected IR rays, the output of OpAmp is high. This output of the op-amp is connected to the reset pin of the 555 timers.

The output of the 555 timers is low when the input voltage applied to pin 4 of the 555 timers is low. When the input voltage at pin 4 is high, the 555 produces a timer frequency that can be adjusted by different value combinations of resistors (R1, R2) and capacitor (C3).

- Constraint current $\rightarrow$ IR receiver receives IR rays $\rightarrow$ Op-amp output high $\rightarrow 555$ RST pin high $\rightarrow$ Speaker produces sound.

- No interrupt $\rightarrow$ IR receiver does not receive rays $\rightarrow$ Op-amp output low $\rightarrow$ RST pin low $\rightarrow \mathrm{R} \rightarrow$ Sound

Many IR sensors are arranged at various locations in the home. The outputs of all these sensors are given to the RESET pin of the common 555 timers. When any sensor detects an outsider, the 555 timer's reset pin goes high and the speaker will produce a sound.

\section{Circuit diagram of the proposed system}

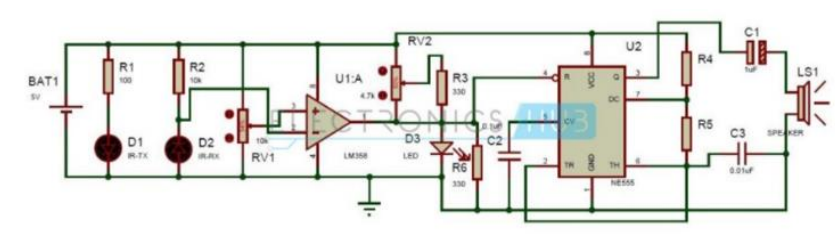

Simulation

The IR transmitter always emits IR (infrared) rays that the IR receiver has to receive. When there is an obstacle in front of the IR sensor, the transmitted IR rays are reflected in the IR receiver. When the IR receiver receives reflected IR rays, the output of Op-Amp is high. This output of the operational amplifier is connected to the RESET pin of the 555 timers.

Constraint current $\rightarrow$ IR receiver receives IR rays $\rightarrow$ Op-amp output high $\rightarrow 555$ RST pin high $\rightarrow$ Speaker produces sound. No interrupt $\rightarrow$ IR receiver does not receive rays $\rightarrow$ Op-amp output low $\rightarrow$ RST pin low $\rightarrow$ No sound

\section{IR SENSOR}

An infrared sensor is an electronic gadget that transmits to detect parts of environmental factors. An IR sensor can determine the warmth of an object such that it recognizes movement. This type of sensor only measures infrared radiation, as opposed to producing what is known as a latent IR sensor. For the most part, in the infrared range, every single article transmits some kind of hot radiation.

Such radiation is undesirable to our eyes, which can be detected by an infrared sensor. The manufacturer is just an IR LED and the identifier is an IR photodiode that is fragile for the very frequency IR light irradiated by the IR LED. At this point when the IR light falls on the photodiode, the protection and yield voltage will change concerning the size of the IR light.

\section{Working principle}

The functioning of an infrared sensor is similar to that of a standard article location sensor. This sensor incorporates an IR LED and IR Photodiode, so connecting these two can be shaped as a photocoupler in any situation. The laws of physics used in this sensor are board radiation, Stephen Boltzman, and Veins displacement.

IR LED is a type of transmitter that emits IR radiation. This LED appears as a standard LED and the radiation it creates is not obvious to the natural eye. Infrared receivers mostly use an infrared transmitter to distinguish radiation. These infrared recipient photodiodes are accessible in structure. IR photodiodes differ from heterogeneous and regular photodiodes because they essentially recognize IR radiation. Different types of infrared collectors mainly depend on voltage, frequency, bundle, and so on. 
Whenever it is used as a mixture of IR transmitter and receiver, at that time the frequency of the collector should increase to the transmitter. However, the transmitter is IR LED, although

The recipient is an IR photodiode. The infrared photodiode is responsible for the infrared light that is produced through an infrared LED. The resistance of the photographic diode and the adjustment in yield voltage is concerning the acquired infrared light. This is the essential working standard of IR sensors.

When the infrared transmitter produces a discharge, at that time it appears on the item and a part of the emission will reflect towards the infrared receiver. The yield of the sensor can be chosen by the IR beneficiary on the force of the reaction.

\section{IC LM358}

This LM358 IC is an incredible, low-power, and simple-to-use double-channel op-amp IC. It is planned and presented by National Semiconductor. It includes two internal frequency compensation, high sum, free op-amp. This IC is planned to work exceptionally to operate from a secluded power supply across a wide range of voltages. The LM358 IC is accessible in a chip-size bundle and the amp in use for this operation includes simple operation amp circuits, DC gain blocks, and transducer intensifiers. The LM358 IC is a decent, standard operating extender and is suitable for your needs. It can deal with $3-32 \mathrm{~V}$ DC supply and sources up to $20 \mathrm{~mA}$ per channel. This operation amp is well suited on the off chance that you need to work two different operation amps to supply a solitary force. It is accessible in an 8-pin DIP bundle

\section{555 TIMER}

The 555 Clock IC was introduced by Signetic Corporation in the year 1970 and Named it SE / NE 555 Clock. It is a solid planning circuit that produces exact and profoundly stable time delays or motions. When contrasted with the uses of an operation amp in similar territories, the 555IC is additionally similarly dependable and is modest. Aside from its applications as a monostable multivibrator and astable multivibrator, a 555 clock can likewise be utilized in dc-dc converters, advanced rationale tests, waveform generators, simple recurrence meters, and tachometers, temperature estimation, and control gadgets, voltage controllers, etc. The timer IC is set up to work in both of the two modes one-shot or monostable or as a free-running or astable multivibrator. The SE 555 can be utilized for temperature runs between $-55^{\circ} \mathrm{C}$ to $125^{\circ}$. The NE 555 can be utilized for a temperature range between $0^{\circ}$ to $70^{\circ} \mathrm{C} . .25^{\circ}$. The NE 555 can be used for a temperature range between $0^{\circ}$ to $70^{\circ} \mathrm{C}$.

\section{POTENTIOMETER}

A potentiometer is a three-terminal resistor with sliding or spindle contact that shapes the movable voltage divider. In case both terminals are used in isolation and the wiper goes as a variable resistor or rheostat. The approximate device called a potentiometer is a voltage divider used to estimate the electric potential (voltage); the clause is the execution of a similar standard.
Potentiometers are commonly used to control electrical equipment, for example, volume control on the sound hardware. A potentiometer worked by an instrument can be used as a position transducer. The potentiometer is used to straighten the critical force (more than one watt) once in a while because the propagation of the force in the potentiometer will be similar to the force in a controlled manner.

\section{CERAMIC CAPACITOR}

A ceramic capacitor is a fixed-respect capacitor where the ceramic material goes as a dielectric. It is made up of at least two exchanging layers of ceramic and a metal layer known as electrodes. The structure of the material is characterized by electrical conductivity and applications accordingly. These capacitors are divided into two application classes:

- Class 1 ceramic capacitors provide high stability and low losses for resonant circuit applications.

- Class 2 ceramic capacitor buffers provide a high degree of productivity for by-pass and coupling applications.

Ceramic capacitors, especially multi-layered ceramic capacitors (MLCC), are the most distributed and used capacitors in electronic gear that connect in about one trillion pieces each year.

\section{ELECTROLYTIC CAPACITOR}

An electrolytic capacitor is an energetic capacitor whose anode or positive plate is made of a metal that shapes a protective oxide layer through an anodization process. This oxide layer goes as the dielectric of the capacitor. The outside of this oxide layer, which fills as the cathode or negative plate of the capacitor, is covered with a strong liquid or gel electrolyte. Because of their extremely dainty dielectric oxide layer and long anode surface, there are much higher capacitance-voltage $(\mathrm{CV})$ items per unit volume than capacitors or film capacitors fused in electrolytic capacitors, thus vast capacitance esteem may be held. There are three groups of electrolytic capacitors: aluminum electrolytic capacitors, tantalum electrolytic capacitors, and niobium electrolytic capacitors.

The large capacitance of electrolytic capacitors makes them particularly appropriate for passing or bypassing lowrepetition signals and removing a lot of energy. They are broadly used for power supply and DC-connected circuits for variable-repetition drives for decompiling or committing shifting, for coupling signals between the intensifier systematics, and to emit energy in the form of a flash lamp

\section{RESULTS AND DISCUSSION}

An outsider alarm is a device that monitors specified areas or areas to detect the presence of suspicious things. The system uses the help of sensors to detect such items. Once found, the system sends an emergency signal to the user or monitor station if the user so desires. The outsider alarm work starts with the sensor. These devices are installed at various locations which 
they monitor in their way. However, they send an emergency signal to the control room when they detect something that exceeds their tolerance level.

The control panel of the system acts as a brain and determines the next stage of action. This can set off the alarm located near the house to alert homeowners and neighbors.

\section{SIGNIFICANCE}

The importance of the project is to decide the presence of an outsider and hence it helps in welfare. It's cheap and affordable. At whatever point the light emission is an obstacle in using any means, it triggers an alert or alarm. This deeply receptive methodology has a low computational condition; It is, therefore, suitable for observation, mechanical applications, and smart environments.

\section{CONCLUSION}

This security framework gives us protection from any wrong activity, theft in our everyday life, thus individuals are presenting them to be protected, safe, and sound. Various electronic security structures can be used at home and other important work sites for security and well-being purposes. It is an unbelievable open gate and there is no practice of saving labor that adds to the waste of electricity. By breaking this structure, theft, robbery and crime can stay away from a huge mass. Maintaining a strategic distance from criminals improves our monetary resources and, as a result, this framework ensures us against all.

\section{FUTURE ENHANCEMENT}

1) We can add a keypad to the hand or disable the alert.

2) We can decide the status of the outsider and later send an SMS to the concerned experts.

3) Inter-availability has different benefits, as well. Not long from now, the outsider alert framework can be directly linked to the criminal information base and can run facial acknowledgment programming.

Combining these and, instead of simply destroying sounds or strobe lights, the framework can address the outsider by Name and immediately send information to experts.

\section{REFERENCES}

1. IEEE Paper Template in A4 (V1) (ijerct.com)

2. Super Sensitive Outsider Alarm Circuit using IR and 555 Timer (electronicshub.org)

3. (PDF) Super Sensitive Outsider Alarm | IJSRD Journal - Academia.edu

4. $\quad$ Super Sensitive Outsider Alarm | CustomWritings

5. Super Sensitive Outsider Alarm Circuit.docx ELECTRONICS PROJECT Super Sensitive Outsider Alarm Circuit Lab Incharge Asad Hassan Submitted by Muhammad | Course Hero
6. Light Sensitive Outsider Alarm Circuit Diagram using 555 IC and LDR (circuitstoday.com)

7. Security alarm - Wikipedia 